\title{
ON STOCHASTIC GENERALIZED FUNCTIONS
}

\author{
PEDRO CATUOGNO AND CHRISTIAN OLIVERA
}

\begin{abstract}
We introduced a new algebra of stochastic generalized functions which contains to the space of stochastic distributions $\mathcal{G}^{*}$, [25]. As an application, we prove existence and uniqueness of the solution of a stochastic Cauchy problem involving singularities.
\end{abstract}

\section{INTRODUCTION}

The algebras of generalized functions were introduced by J. F. Colombeau [8] and has been studied and developed by many authors (see [7], 8], [12] and 22] and references). These algebras of generalized functions contain the Schwartz distributions, thus one can deal with its multiplication and other types of nonlinearities. Many applications have been carried out in various fields of mathematics such as partial differential equations, Lie analysis, local and microlocal analysis, probability theory, differential geometry. In particular, the algebras of generalized functions found applications in non-linear partial differential equations (7], 8], 12] and [22]), where the classical distributional methods are limited by the impossibility to consistently define an intrinsic product of distributions [27.

In recent years stochastic partial differential equations have been studied actively. Often, solutions to such equations do not exist in the usual sense, rather they are generalized functions. It is possible consider the solutions as generalized functions in the space-time variables or as generalized stochastic functions which are point-wise defined in space and time. In this work we take the latter approach, more precisely we consider stochastic process in algebras of generalized functions, following to F. Russo and their coauthors (see for instance [2], [3] , [23] and [26].

Key words and phrases. White noise, Wick product, Generalized functions, Colombeau Algebras, parabolic equation.

2000 Mathematics Subject Classification. Primary: 46F30, 60G20 ; Secondary: $60 B 99$.

Research partially supported by FAPESP 02/10246-2, 2007/54740-4 and CNPQ 302704/2008-6. 
We considerer the following stochastic Cauchy problem,

$$
\left\{\begin{array}{l}
u_{t}=L u+u W(t, x) \\
u(0, x)=f(x)
\end{array}\right.
$$

where the product is taken in the usual form, $L$ is an uniformly elliptic partial differential operator, $f \in C_{b}^{2}\left(\mathbb{R}^{l}\right)$ and $W$ is a space-time white noise [14]. There are a great interest into solve the problem (11) (see for instance [10, [15], 19], 20, [17, 21, 24 and 30]). We observe that the properties of the solutions (Itô-Stratonovich) of (11) depend crucially on the noise type and its dimension (see [17] and [30]).

The aim of this article is solve the stochastic Cauchy problem (1) without restriction on the dimension of the white noise $W$. Our approach transfer the generalized nature of the solutions to the stochastic component (see [15] for more details). It is thus necessary to introduce random variables with values in algebras of generalized functions. The fundamental idea is to introduce a new algebra of generalized functions of Colombeau type in the context of the Hida stochastic distributions. This algebra of generalized functions contains the stochastic distributions $\mathcal{G}^{*}$ introduced by J. Potthoff and M. Timpel in [25]. In this context and under general assumptions we show existence and uniqueness for the stochastic Cauchy problem (1). Furthermore, others aspects of these generalized functions are studied.

The plan of exposition is as follows: in section 2, we provide some basic concepts on classical white noise theory, these are, Wiener-Itô chaos expansions, stochastic distributions and Wick products. In section 3, we introduce three definitions of products of stochastic distributions and we study some properties. In section 4 , we give a new algebra of stochastic generalized functions $\mathcal{G}$, this algebra contains to the stochastic distributions $\mathcal{G}^{*}$ ( see [25]) and extends the product in the test space $\mathcal{G}$. Moreover, we study its elementary properties and we show that the

symmetric product of stochastic distributions, defined in section 3, is associated with the product in $\mathcal{G}$. Finally, in section 5, we study a stochastic Cauchy problem with space-time depend noise white in the context of these generalized functions.

\section{White Noise Theory}

2.1. Preliminaries. Let $\mathcal{S}\left(\mathbb{R}^{d}\right)$ be the space of infinitely differentiable functions from $\mathbb{R}^{d}$ to $\mathbb{R}(\mathbb{C})$ which together with all its derivatives are of rapidly decreasing. We consider $\mathcal{S}\left(\mathbb{R}^{d}\right)$ with the topology given by the family of seminorms

$$
\|f\|_{\alpha, \beta}=\sup \left\{\left|x^{\alpha} D^{\beta} f(x)\right|: x \in \mathbb{R}^{d}\right\}
$$


where $\alpha, \beta \in \mathbb{N}_{0}^{d}$. It follows that $\mathcal{S}\left(\mathbb{R}^{d}\right)$ is a nuclear space. The Schwartz space of tempered distributions is the dual space $\mathcal{S}^{\prime}\left(\mathbb{R}^{d}\right)$.

We shall summarize the definitions and basic properties of Hermite polynomials and Hermite functions. The $j$-th Hermite polynomial, denoted by $h_{j}$, is defined to be

$$
h_{j}(x)=(-1)^{n} e^{\frac{x^{2}}{2}} \frac{d^{j}}{d x^{j}} e^{-\frac{x^{2}}{2}}
$$

for $j \in \mathbb{N}_{0}$.

We observe that

$$
h_{j}(x)=2^{-\frac{j}{2}} \sum_{k=0}^{[j / 2]} \frac{(-1)^{k} n !(\sqrt{2} x)^{j-2 k}}{k !(j-2 k) !} .
$$

The $j$-th Hermite function $\eta_{j}$ is given by

$$
\eta_{j}(x)=(\sqrt{2 \pi} n !)^{-\frac{1}{2}} e^{-\frac{1}{4} x^{2}} h_{j}(x)
$$

for $j \in \mathbb{N}_{0}$.

The following properties of the Hermite functions will be extremely useful.

- $\left\{\eta_{j}: j \in \mathbb{N}_{0}\right\}$ is an orthonormal basis of $L^{2}(\mathbb{R})$,

- $\sup \left\{\left|\eta_{j}(x)\right|: x \in \mathbb{R}\right\}=O\left(j^{-\frac{1}{4}}\right)$,

- The $\eta_{j}$ are eigenfunctions of the number operator $N+1=$ $\frac{1}{2}\left(-\frac{d^{2}}{d x^{2}}+x^{2}+1\right)$

$$
(N+1) \eta_{j}=(j+1) \eta_{j}
$$

for all $j \in \mathbb{N}_{0}$.

The $\alpha$-th Hermite function $\eta_{\alpha}: \mathbb{R}^{d} \rightarrow \mathbb{R}\left(\alpha \in \mathbb{N}_{0}^{d}\right)$ is given by

$$
\eta_{\alpha}(x)=\prod_{i=1}^{d} \eta_{\alpha_{i}}\left(x_{i}\right) .
$$

It follows that $\left\{\eta_{\alpha}: \alpha \in \mathbb{N}_{0}^{d}\right\}$ is an orthonormal basis of $L^{2}\left(\mathbb{R}^{d}\right)$.

Let us denote by $(N+1)_{d}=(N+1)^{\otimes d}$, the differential operator

$$
(N+1)^{\otimes d}=\frac{1}{2}\left(-\frac{\partial^{2}}{\partial x_{d}^{2}}+x_{d}^{2}+1\right) \cdots \frac{1}{2}\left(-\frac{\partial^{2}}{\partial x_{1}^{2}}+x_{1}^{2}+1\right) .
$$

It is easy to check that for $\alpha, \beta \in \mathbb{N}_{0}^{d}$,

$$
(N+1)_{d}^{\beta}\left(\eta_{\alpha}\right)=(\alpha+1)^{\beta} \eta_{\alpha}
$$

where $(\alpha+1)^{\beta}=\prod_{i=1}^{d}\left(1+\alpha_{i}\right)^{\beta_{i}}$. 
The topology of $\mathcal{S}\left(\mathbb{R}^{d}\right)$ has an alternative description in terms of the family of norms $\left\{|\cdot|_{\beta}: \beta \in \mathbb{N}_{0}^{d}\right\}$. The norm $|\cdot|_{\beta}$ is given by

$$
|\varphi|_{\beta}^{2}:=\left|(N+1)_{d}^{\beta}(\varphi)\right|_{0}^{2}=\sum_{\alpha \in \mathbb{N}_{0}^{d}}(\alpha+1)^{2 \beta}<\varphi, \eta_{\alpha}>^{2},
$$

where $\left\langle\varphi, \eta_{\alpha}\right\rangle=\int \varphi(x) \eta_{\alpha}(x) d x$ are the Fourier-Hermite coefficients of the expansion of $\varphi$.

It follows easily that the seminorm families $\left\{|\cdot|_{\beta}: \beta \in \mathbb{N}_{0}^{d}\right\}$ and $\left\{\|\cdot\|_{\alpha, \beta}\right.$ : $\left.\alpha, \beta \in \mathbb{N}_{0}^{d}\right\}$ are equivalent on $\mathcal{S}\left(\mathbb{R}^{d}\right)$.

The Hermite representation theorem for $\mathcal{S}\left(\mathbb{R}^{d}\right)\left(\mathcal{S}^{\prime}\left(\mathbb{R}^{d}\right)\right)$ states a topological isomorphism from $\mathcal{S}\left(\mathbb{R}^{d}\right)\left(\mathcal{S}^{\prime}\left(\mathbb{R}^{d}\right)\right)$ onto the space of sequences $\mathbf{s}_{d}\left(\mathbf{s}_{d}^{\prime}\right)$.

Let $\mathbf{s}_{d}$ be the space of sequences

$$
\left.\mathbf{s}_{d}=\left\{\left(a_{\beta}\right) \in \ell^{2}\left(\mathbb{N}^{d}\right)\right): \sum_{\beta}(\beta+1)^{2 \alpha}\left|a_{\beta}\right|^{2}<\infty, \text { for all } \alpha \in \mathbb{N}_{0}^{d}\right\} .
$$

The space $\mathbf{s}$ is a locally convex space with the sequence of norms

$$
\left|\left(a_{\beta}\right)\right|_{\alpha}=\left(\sum_{\beta}(\beta+1)^{2 \alpha}\left|a_{\alpha}\right|^{2}\right)^{\frac{1}{2}}
$$

The topological dual space to $\mathbf{s}_{d}$, denoted by $\mathbf{s}_{d}^{\prime}$, is given by

$$
\mathbf{s}_{d}^{\prime}=\left\{\left(b_{\beta}\right) \text { : for some }(C, \alpha) \in \mathbb{R} \times \mathbb{N}_{0}^{d},\left|b_{\beta}\right| \leq C(\beta+1)^{\alpha} \text { for all } \beta\right\},
$$

and the natural pairing of elements from $\mathbf{s}_{d}$ and $\mathbf{s}_{d}^{\prime}$, denoted by $\langle\cdot, \cdot\rangle$, is given by

$$
\left\langle\left(b_{\beta}\right),\left(a_{\beta}\right)\right\rangle=\sum_{\beta} b_{\beta} a_{\beta}
$$

for $\left(b_{\beta}\right) \in \mathbf{s}_{d}^{\prime}$ and $\left(a_{\beta}\right) \in \mathbf{s}_{d}$.

It is clear that $\mathbf{s}_{d}^{\prime}$ is an algebra with the pointwise operations:

$$
\begin{aligned}
\left(b_{\beta}\right)+\left(b_{\beta}^{\prime}\right) & =\left(b_{\beta}+b_{\beta}^{\prime}\right) \\
\left(b_{\beta}\right) \cdot\left(b_{\beta}^{\prime}\right) & =\left(b_{\beta} b_{\beta}^{\prime}\right),
\end{aligned}
$$

and $\mathbf{s}_{d}$ is an ideal of $\mathbf{s}_{d}^{\prime}$.

The relation between $\mathbf{s}_{d}\left(\mathbf{s}_{d}^{\prime}\right)$ and $\mathcal{S}\left(\mathbb{R}^{d}\right)\left(\mathcal{S}^{\prime}\left(\mathbb{R}^{d}\right)\right)$ is induced by the Hermite functions, via the Fourier-Hermite coefficients.

Theorem 1. N-representation theorem for $\mathcal{S}\left(\mathbb{R}^{d}\right)$ and $\mathcal{S}^{\prime}\left(\mathbb{R}^{d}\right)$ :

a) Let $\mathbf{h}: \mathcal{S}\left(\mathbb{R}^{d}\right) \rightarrow \mathbf{s}_{d}$ be the application

$$
\mathbf{h}(\varphi)=\left(<\varphi, \eta_{\beta}>\right) .
$$

Then $\mathbf{h}$ is a topological isomorphism. Moreover,

$$
\|\mathbf{h}(\varphi)\|_{\beta}=\|\varphi\|_{\beta}
$$


for all $\varphi \in \mathcal{S}\left(\mathbb{R}^{d}\right)$.

b) Let $\mathbf{H}: \mathcal{S}^{\prime}\left(\mathbb{R}^{d}\right) \rightarrow \mathbf{s}_{d}^{\prime}$ be the application $\mathbf{H}(T)=\left(T\left(\eta_{\beta}\right)\right)$. Then $\mathbf{H}$ is a topological isomorphism. Moreover, if $T \in \mathcal{S}^{\prime}\left(\mathbb{R}^{d}\right)$ we have that

$$
T=\sum_{\beta} T\left(\eta_{\beta}\right) \eta_{\beta}
$$

in the weak sense and for all $\varphi \in \mathcal{S}\left(\mathbb{R}^{d}\right)$,

$$
T(\varphi)=\langle\mathbf{H}(T), \mathbf{h}(\varphi)\rangle .
$$

Proof. See for instance, M. Reed and B. Simon [28] pp. 143.

We say that the sequences $\mathbf{h}(\varphi)$ and $\mathbf{H}(T)$ are the Hermite coefficients of the tempered function $\varphi$ and the tempered distribution $T$, respectively.

2.2. White noise and chaos expansions. In this subsection we briefly recall some of the basic concepts and results of White noise analysis. Our presentation and notation will follow [14] and [15]. According to the Bochner-Minlos theorem, there exits an unique probability $\mu$ on $\mathcal{B}_{d}$ the Borel $\sigma$-field of $\mathcal{S}^{\prime}\left(\mathbb{R}^{d}\right)$ such that for each $\phi \in \mathcal{S}\left(\mathbb{R}^{d}\right)$,

$$
\int_{\mathcal{S}^{\prime}\left(\mathbb{R}^{d}\right)} e^{i(\omega, \phi)} d \mu(\omega)=e^{-\frac{1}{2}|\phi|_{L^{2}\left(\mathbb{R}^{d}\right)}^{2}}
$$

The probability space $\left(\mathcal{S}^{\prime}\left(\mathbb{R}^{d}\right), \mathcal{B}_{d}, \mu\right)$ is then called the White noise probability space. We will denote by $\left(L^{2}\right)$ the space $L^{2}\left(\mathcal{S}^{\prime}\left(\mathbb{R}^{d}\right), \mathcal{B}, \mu\right)$.

Let $\left\{\alpha^{j}: j \in \mathbb{N}_{0}\right\}$ be an enumeration of the set of multi-indices $\{\alpha: \alpha \in$ $\left.\mathbb{N}_{0}^{d}\right\}$. We can assume that this enumeration has the following property: If $k<l$ then $\alpha_{1}^{k}+\ldots+\alpha_{d}^{k} \leq \alpha_{1}^{l}+\ldots+\alpha_{d}^{l}$ (see [15] pag. 19 for details). It is clear that $\left\{\eta_{\alpha}: \alpha \in \mathbb{N}_{0}^{d}\right\}$ can be enumerated by $\left\{\eta_{j}: j \in \mathbb{N}_{0}\right\}$. We denote by $\mathcal{J}$ the set of sequences of nonnegative integers with compact support.

The $\alpha$-th Hermite polynomial $H_{\alpha}: \mathcal{S}^{\prime}\left(\mathbb{R}^{d}\right) \rightarrow \mathbb{R}(\alpha \in \mathcal{J})$ is given by

$$
H_{\alpha}(\omega)=\prod_{j=1}^{\infty} h_{\alpha_{j}}\left(<\omega, \eta_{j}>\right)
$$

It follows that $\left\{H_{\alpha}: \alpha \in \mathcal{J}\right\}$ is an orthogonal basis of $\left(L^{2}\right)$ and $\left\|H_{\alpha}\right\|^{2}=$ $\alpha$ !. The Wiener-Itô chaos expansion theorem says that if $f \in\left(L^{2}\right)$, then there exists an unique sequence of functions $f_{n} \in L^{2}\left(\mathbb{R}^{d}\right)^{\hat{\otimes} n}$ such that

$$
f(\omega)=\sum_{n=0}^{\infty} I_{n}\left(f_{n}\right)(\omega)
$$


where $I_{n}: L^{2}\left(\mathbb{R}^{d}\right)^{\hat{\otimes} n} \rightarrow\left(L^{2}\right)$ is the multiple Wiener integral of order $n$. Moreover, we have the isometry

$$
\mathbb{E}\left[f^{2}\right]=\sum_{n=0}^{\infty} n !\left|f_{n}\right|_{0}^{2} .
$$

where we denote by $|\cdot|_{0}$ the norm $|\cdot|_{L^{2}\left(\mathbb{R}^{d}\right)^{\hat{\otimes}} n}$.

Let $f \in \mathcal{S}(\mathbb{R})$, from (5) for $d=1$ we have that $\langle\cdot, f\rangle$ is a Gaussian random variable with expectation 0 and variance $|f|_{0}$. We observe that for $t \in \mathbb{R}$,

$$
B(t):=<\cdot, 1_{[0, t]}>
$$

is a Brownian motion. Moreover, if $f \in L^{2}(\mathbb{R})$ we have that

$$
<\cdot f>=I_{1}(f) .
$$

2.3. Stochastic distributions. The space of stochastic distributions (also called of space of regular Hida distributions) $\mathcal{G}^{*}$ has been introduced by J. Potthoff and M. Timpel in [25]. Their development was motivated by stochastic differential equations of Wick type and its applications, see for instance [1], [5], 6] and [13].

Let $N$ be the Ornstein-Uhlenbeck operator on $\left(L^{2}\right)$, we recall that

$$
N\left(I_{n}(f)\right)=n I_{n}(f)
$$

for all $n \in \mathbb{N}_{0}$ and $f \in L^{2}\left(\mathbb{R}^{d}\right)^{\hat{\otimes} n}$.

Let us denote by $\mathcal{G}_{\sqrt{ }}\left(p \in \mathbb{N}_{0}\right)$ the $\left(L^{2}\right)$-domain of $\exp (p N)$. It is clear that $\mathcal{G}_{\sqrt{ }}$ with the norm

$$
\|F\|_{p}^{2}:=\|\exp (p N) F\|_{0}^{2}=\sum_{n=0}^{\infty} e^{2 p n} n !\left|F_{n}\right|_{0}^{2}
$$

is a Hilbert space.

Let $\mathcal{G}$ be the projective limit of the family $\left\{\mathcal{G}_{\sqrt{ }}, p \geq 0\right\}$ and $\mathcal{G}^{*}$ be its dual. It follows that $\mathcal{G}^{*}$ is the inductive limit of the family $\left\{\mathcal{G}_{-}, p \geq 0\right\}$, where $\mathcal{G}_{-}$is the dual of $\mathcal{G}_{\sqrt{ }}$. In this way we obtained the Gelfand triple

$$
\mathcal{G} \subset\left(L^{2}\right) \subset \mathcal{G}^{*} .
$$

We observe that the duality in this Gelfand triple is given by

$$
\ll F, f \gg=\sum_{n=0}^{\infty} n !<F_{n}, f_{n}>
$$

where $f=\sum_{n=0}^{\infty} I_{n}\left(f_{n}\right) \in \mathcal{G}$ and $F=\sum_{n=0}^{\infty} I_{n}\left(F_{n}\right) \in \mathcal{G}^{*}$. 
Example 1. The Brownian motion $B(t)=<\cdot, 1_{[0, t]}>$ is an element of $\mathcal{G}$. J. Potthof and M. Timpel [25] showed that for $x \in \mathbb{R}$ the Donsker delta function $\delta_{x} \circ B(t)$ belong to $\mathcal{G}^{*}$.

Let $F \in \mathcal{G}^{*}$ with Wiener-Itô expansion $\sum_{i=0}^{\infty} I_{n}\left(F_{n}\right)$. The $S$-transform of $F \in \mathcal{G}^{*}$, denoted by $S(F)$, is the function from $\mathcal{S}\left(\mathbb{R}^{d}\right)$ to $\mathbb{C}$ given by

$$
S(F)(\varphi)=\sum_{n=0}^{\infty}<F_{n}, \varphi^{\otimes n}>.
$$

The Wick product $F \diamond G$ of $F$ and $G$ in $\mathcal{G}^{*}$, is given by $S^{-1}(S(F) S(G)$ ). This product is well defined and continuous from $\mathcal{G}^{*} \times \mathcal{G}^{*}$ to $\mathcal{G}^{*}$, see [6], [16] and [25].

We observe that the Wick product is associative, commutative and $\mathcal{G}$ and $\mathcal{G}^{*}$ are algebras respect to the Wick product. An easy computation shows that if $F=\sum_{i=0}^{\infty} I_{i}\left(F_{i}\right)$ and $G=\sum_{i=0}^{\infty} I_{i}\left(G_{i}\right)$ belong to $\mathcal{G}^{*}$ then the Wiener-Itô coefficients of the Wick product $F \diamond G=\sum_{i=0}^{\infty} I_{i}\left(H_{i}\right)$ verify that

$$
H_{i}=\sum_{i=m+n} F_{n} \hat{\otimes} G_{m}
$$

The Wick exponential of $f \in L^{2}\left(\mathbb{R}^{d}\right)$, denoted by $e^{:<\cdot, f>}$, is the element of $\left(L^{2}\right)$ defined by

$$
\sum_{m=0}^{\infty} \frac{1}{m !} I_{m}\left(f^{\otimes^{m}}\right)=e^{<\cdot, f>-\frac{1}{2}|f|_{0}^{2}} .
$$

We observe the following useful identity

$$
\left\|e^{:<\cdot, f>}\right\|_{p}^{2}=\sum_{m=0}^{\infty} \frac{e^{2 p m}}{m !}|f|_{0}^{2 m}=e^{e^{2 p}|f|_{0}^{2}} .
$$

The space $\mathcal{G}$ is an algebra for the pointwise multiplication and this multiplication is continuous from $\mathcal{G} \times \mathcal{G}$ into $\mathcal{G}$, see Theorem 2.5 of [25] $(d=1)$ and [6] for the general case. In particular, for all $m \in \mathbb{N}_{0}$ there exists $r, s \in \mathbb{N}_{0}$ and a constant $C_{m}>0$ such that

$$
\|\varphi \psi\|_{m} \leq C_{m}\|\varphi\|_{r}\|\psi\|_{s}
$$

for all $\varphi, \psi \in \mathcal{G}$.

Thus we can define the product of a distribution by a test function.

Definition 1. Let $\varphi \in \mathcal{G}$ and $F \in \mathcal{G}^{*}$. We define $\varphi F \in \mathcal{G}^{*}$ by

$$
\ll \varphi F, \psi \gg=\ll F, \varphi \psi \gg
$$

for all $\psi \in \mathcal{G}$. 
It is clear from (9) that $\varphi F$ is well defined.

Let $\varphi=\sum_{n=0}^{\infty} I_{n}\left(\varphi_{n}\right) \in \mathcal{G}$ and $F=\sum_{m=0}^{\infty} I_{m}\left(F_{m}\right) \in \mathcal{G}^{*}$. Then $\varphi F=$ $\sum_{j=0}^{\infty} I_{j}\left(H_{j}\right)$ where

$$
H_{j}=\sum_{j=m+n} \sum_{k=0}^{m \wedge n} k !\left(\begin{array}{c}
n+k \\
k
\end{array}\right)\left(\begin{array}{c}
m+k \\
k
\end{array}\right) \varphi_{n+k} \hat{\otimes}_{k} F_{m+k}
$$

Here $\varphi_{n} \hat{\otimes}_{k} F_{m}$ is the symmetrization of $\varphi_{n} \otimes_{k} F_{m}$. See for instance [16] pag. 70 .

\section{Distributional products via approximation in ChaOs}

In this section, strongly inspired in [9], we define three new products of stochastic distributions. A possible approach to define a product of a pair of distributions is approximate one of them by test functions, multiply this approximation by the other distribution, and pass to a limit. In the case of the sequential approach (see [4] pp 242, [22]) the approximation is done by convolution with $\delta$-sequences, in this work we propose take the approximation given by the Wiener-Itô chaos expansion of the distribution.

In order to define the chaos approximation we consider the projections $\Pi_{m}: \mathcal{G}^{*} \rightarrow \mathcal{G}\left(m \in \mathbb{N}_{0}\right)$. These projections are defined by

$$
\Pi_{m} F=\sum_{n=0}^{m} I_{n}\left(F_{n}\right)
$$

where $F=\sum_{n=0}^{\infty} I_{n}\left(F_{n}\right) \in \mathcal{G}^{*}$.

Proposition 1. a) Let $f=\sum_{n=0}^{\infty} I_{n}\left(f_{n}\right) \in \mathcal{G}$. Then

$$
\lim _{m \rightarrow \infty} \Pi_{m} f=f .
$$

b) Let $F=\sum_{n=0}^{\infty} I_{n}\left(F_{n}\right) \in \mathcal{G}^{*}$. Then

$$
\lim _{m \rightarrow \infty} \Pi_{m} F=F .
$$

Proof. a) Let $p>0$ and $\varepsilon>0$. Since $f \in \mathcal{G}$, there exists $m_{0} \in \mathbb{N}_{0}$ such that

$$
\sum_{n=m+1}^{\infty} n ! e^{2 p n}\left|f_{n}\right|_{0}^{2}<\varepsilon^{2}
$$

for $m \geq m_{0}$. 
Then

$$
\begin{aligned}
\left\|\Pi_{m} f-f\right\|_{p}^{2} & =\sum_{n=m+1}^{\infty} n ! e^{2 p n}\left|f_{n}\right|_{0}^{2} \\
& <\varepsilon^{2}
\end{aligned}
$$

for $m \geq m_{0}$. We conclude that $\lim _{m \rightarrow \infty} \Pi_{m} f=f$.

b) Let $\varepsilon>0$. Since $F \in \mathcal{G}^{*}$ there exists $q \in \mathbb{N}_{0}$ such that

$$
\|F\|_{-q}^{2}=\sum_{n=0}^{\infty} n ! e^{-2 q n}\left|F_{n}\right|_{0}^{2}<\infty .
$$

Then there exists $m_{0} \in \mathbb{N}_{0}$ such that

$$
\sum_{n=m+1}^{\infty} n ! e^{-2 q n}\left|F_{n}\right|_{0}^{2}<\varepsilon^{2} .
$$

for $m \geq m_{0}$.

Thus

$$
\begin{aligned}
\left\|\Pi_{m} F-F\right\|_{-q}^{2} & =\sum_{n=m+1}^{\infty} n ! e^{-2 q n}\left|F_{n}\right|_{0}^{2} \\
& <\varepsilon^{2}
\end{aligned}
$$

for $m \geq m_{0}$.

Now, we introduce the following products of stochastic distributions.

Definition 2. Let $F$ and $G$ be stochastic distributions. The products $F \bullet \bullet_{r} G, F \bullet G$ and $F \bullet G$ are, by definition,

$$
\begin{gathered}
F \bullet_{r} G=\lim _{m \rightarrow \infty} F\left(\Pi_{m} G\right) \\
F \bullet l=\lim _{m \rightarrow \infty}\left(\Pi_{m} F\right) G \\
F \bullet G=\lim _{m \rightarrow \infty}\left(\Pi_{m} F\right)\left(\Pi_{m} G\right) .
\end{gathered}
$$

where the limits are taking in the weak sense.

Proposition 2. The products (11), (12) and (13) extend the product of elements of $\mathcal{G}$ and $\mathcal{G}^{*}$.

Proof. It follows from the bi-continuity of the product of elements of $\mathcal{G}$ and $\mathcal{G}^{*}$. 
We recall that two elements $F=\sum_{n=0}^{\infty} I_{n}\left(F_{n}\right)$ and $G=\sum_{n=0}^{\infty} I_{n}\left(G_{n}\right)$ of $\mathcal{G}^{*}$ are strongly independent (see F. Benth and J. Potthoff [5]), if there exists two intervals $I_{F}$ and $I_{G}$ whose intersection has Lebesgue measure zero such that $\operatorname{supp}\left(F_{n}\right) \subseteq I_{F}^{n}$ and $\operatorname{supp}\left(G_{n}\right) \subseteq I_{G}^{n}$ for all $n \in \mathbb{N}$.

Proposition 3. Let $F$ and $G$ be strongly independent in $\mathcal{G}^{*}$. Then

$$
F \bullet G=F \diamond G .
$$

Proof. Let $F=\sum_{n=0}^{\infty} I_{n}\left(F_{n}\right)$ and $G=\sum_{k=0}^{\infty} I_{k}\left(G_{k}\right)$ be strongly independent in $\mathcal{G}^{*}$. It is clear that $\lim _{m \rightarrow \infty}\left(\Pi_{m} F\right)\left(\Pi_{m} G\right)$ is equal to

$$
\lim _{m \rightarrow \infty} \sum_{n=0}^{m} \sum_{k=0}^{m} \sum_{j=0}^{n \wedge k} k !\left(\begin{array}{l}
n \\
j
\end{array}\right)\left(\begin{array}{l}
k \\
j
\end{array}\right) I_{n+k-2 j}\left(F_{n} \hat{\otimes}_{j} G_{k}\right) .
$$

Since $F$ and $G$ are strongly independent in $\mathcal{G}^{*}$,

$$
\sum_{j=0}^{n \wedge k} k !\left(\begin{array}{l}
n \\
j
\end{array}\right)\left(\begin{array}{l}
k \\
j
\end{array}\right) I_{n+k-2 j}\left(F_{n} \hat{\otimes}_{j} G_{k}\right)=I_{n+k}\left(F_{n} \hat{\otimes} G_{k}\right) .
$$

Combining the above equalities we conclude that

$$
\begin{aligned}
F \bullet G & =\lim _{m \rightarrow \infty}\left(\Pi_{m} F\right)\left(\Pi_{m} G\right) \\
& =\lim _{m \rightarrow \infty} \sum_{n=0}^{m} \sum_{k=0}^{m} I_{n+k}\left(F_{n} \hat{\otimes} G_{k}\right) \\
& =F \diamond G .
\end{aligned}
$$

\section{Stochastic Generalized FunCtions}

In order to introduce an algebra of Colombeau type such that include the stochastic distributions we consider $\mathcal{G}^{\mathbb{N}_{0}}$ the space of sequences of $\mathcal{G}$. It is clear that $\mathcal{G}^{\mathbb{N}_{0}}$ has the structure of an associative, commutative algebra with the natural operations:

$$
\begin{aligned}
\left(\varphi_{n}\right)+\left(\psi_{n}\right) & =\left(\varphi_{n}+\psi_{n}\right) \\
a\left(\varphi_{n}\right) & =\left(a \varphi_{n}\right) \\
\left(\varphi_{n}\right) \cdot\left(\psi_{n}\right) & =\left(\varphi_{n} \psi_{n}\right)
\end{aligned}
$$

where $\left(\varphi_{n}\right)$ and $\left(\psi_{n}\right)$ are in $\mathcal{G}$ and $a \in \mathbb{R}$.

We introduce the following sequence spaces. Let $\mathbf{e}$ be given by

$$
\mathbf{e}=\left\{\left(a_{n}\right) \in \ell^{2}: \lim _{n \rightarrow \infty} e^{n m} a_{n}=0, \text { for all } m \in \mathbb{N}_{0}\right\} .
$$


We consider e equipped with the sequence of norms $\left(m \in \mathbb{N}_{0}\right)$

$$
\left|\left(a_{n}\right)\right|_{m}=\left(\sum_{n=0}^{\infty} e^{2 n m}\left|a_{n}\right|^{2}\right)^{\frac{1}{2}}
$$

or with the equivalent sequence of norms

$$
\left|\left(a_{n}\right)\right|_{m, \infty}=\sup _{n} e^{n m}\left|a_{n}\right| .
$$

It is clear that $\mathbf{e}$ is a locally convex space.

Let us denote by $\mathbf{e}^{\prime}$ the topological dual space to e (see [29]). It follows that $\mathbf{e}^{\prime}$ is given by

$$
\mathbf{e}^{\prime}=\left\{\left(b_{n}\right) \text { : for some }(C, m) \in \mathbb{R} \times \mathbb{N}_{0},\left|b_{n}\right| \leq C e^{2 n m} \text { for all } n\right\} .
$$

The natural pairing of elements from $\mathbf{e}$ and $\mathbf{e}^{\prime}$, denoted by $\langle\cdot, \cdot\rangle$, is given by

$$
\left\langle\left(b_{n}\right),\left(a_{n}\right)\right\rangle=\sum_{n=0}^{\infty} b_{n} a_{n}
$$

for $\left(b_{n}\right) \in \mathbf{e}^{\prime}$ and $\left(a_{n}\right) \in \mathbf{e}$.

We observe that $\mathbf{e}^{\prime}$ is an algebra with the pointwise operations and $\mathbf{e}$ is an ideal.

Definition 3. Let

$$
G_{\mathbf{e}}=\left\{\left(\varphi_{m}\right) \in \mathcal{G}^{\mathbb{N}_{0}}: \forall p \in \mathbb{N}_{0}\left(\left\|\varphi_{m}\right\|_{p}\right) \in \mathbf{e}\right\}
$$

and

$$
G_{\mathbf{e}^{\prime}}=\left\{\left(\varphi_{m}\right) \in \mathcal{G}^{\mathbb{N}_{0}}: \forall p \in \mathbb{N}_{0}\left(\left\|\varphi_{m}\right\|_{p}\right) \in \mathbf{e}^{\prime}\right\} .
$$

Lemma 1. $G_{\mathbf{e}^{\prime}}$ is a subalgebra of $\mathcal{G}^{\mathbb{N}_{0}}$ and $G_{\mathbf{e}}$ is an ideal of $G_{\mathbf{e}^{\prime}}$.

Proof. Let $\left(\varphi_{n}\right),\left(\psi_{n}\right) \in G_{\mathbf{e}^{\prime}}$ and $m \in \mathbb{N}_{0}$. From (9) it follows that there exists $r, s \in \mathbb{N}_{0}$ and a constant $C_{m}>0$ such that

$$
\left\|\varphi_{n} \psi_{n}\right\|_{m} \leq C_{m}\left\|\varphi_{n}\right\|_{r}\left\|\psi_{n}\right\|_{s}
$$

By definition, there exists constants $D, E>0$ and $p, q \in \mathbb{N}_{0}$ such that

$$
\begin{aligned}
& \left\|\varphi_{n}\right\|_{r} \leq D e^{n p} \\
& \left\|\psi_{n}\right\|_{s} \leq E e^{n q} .
\end{aligned}
$$

Combining these inequalities, we obtain

$$
\left\|\varphi_{n} \psi_{n}\right\|_{m} \leq C_{m} D E e^{n(p+q)} .
$$

This proves that $\left(\left\|\varphi_{n} \psi_{n}\right\|_{m}\right) \in \mathbf{e}^{\prime}$, thus $\left(\varphi_{n}\right) \cdot\left(\psi_{n}\right) \in G_{\mathbf{e}^{\prime}}$. 
It remains to prove that $G_{\mathbf{e}}$ is an ideal of $G_{\mathbf{e}^{\prime}}$. Let $\left(\varphi_{n}\right) \in G_{\mathbf{e}^{\prime}},\left(\psi_{n}\right) \in G_{\mathbf{e}}$ and $m \in \mathbb{N}_{0}$. By definitions, we have that for each $r \in \mathbb{N}_{0}$ there exists a constant $D>0$ and $p \in \mathbb{N}_{0}$ such that

$$
\left\|\varphi_{n}\right\|_{r} \leq D e^{n p}
$$

and for all $s, l \in \mathbb{N}_{0}$,

$$
\left|\left(\left\|\psi_{n}\right\|_{s}\right)\right|_{l}^{2}=\sum_{n=0}^{\infty} e^{2 l p}\left\|\psi_{n}\right\|_{s}^{2}<\infty .
$$

Combining the inequality (9) with the above equations we obtain

$$
\begin{aligned}
\left|\left(\left\|\varphi_{n} \psi_{n}\right\|_{m}\right)\right|_{l}^{2} & =\sum_{n=0}^{\infty} e^{2 n l}\left\|\varphi_{n} \psi_{n}\right\|_{m}^{2} \\
& \leq C_{m}^{2} \sum_{n=0}^{\infty} e^{2 n l}\left\|\varphi_{n}\right\|_{r}^{2}\left\|\psi_{n}\right\|_{s}^{2} \\
& \leq C_{m}^{2} D^{2} \sum_{n=0}^{\infty} e^{2 n(l+p)}\left\|\psi_{n}\right\|_{s}^{2} \\
& <\infty .
\end{aligned}
$$

We have proved that $\left(\left\|\varphi_{n} \psi_{n}\right\|_{m}\right) \in \mathbf{e}$, for all $m \in \mathbb{N}_{0}$, this is $\left(\varphi_{n}\right) \cdot\left(\psi_{n}\right) \in$ $G_{\mathbf{e}}$.

Proposition 4. Let $F \in \mathcal{G}^{*}$. Then $\left(F_{m}\right) \in G_{\mathbf{e}^{\prime}}$, where $F_{m}=\Pi_{m} F$.

Proof. Since $F=\sum_{n=0}^{\infty} I_{n}\left(F_{n}\right) \in \mathcal{G}^{*}$, there exists $q \in \mathbb{N}_{0}$ such that

$$
\|F\|_{-q}^{2}=\sum_{n=0}^{\infty} n ! e^{-2 q n}\left|F_{n}\right|_{0}^{2}<\infty .
$$

Then

$$
\begin{aligned}
\left\|F_{m}\right\|_{p}^{2} & =\sum_{n=0}^{m} n ! e^{2 p n}\left|F_{n}\right|_{0}^{2} \\
& \leq e^{(2 p+2 q) m}\|F\|_{-q}^{2}
\end{aligned}
$$

for all $p \in \mathbb{N}_{0}$. This proves that $\left(F_{m}\right) \in G_{\mathbf{e}^{\prime}}$.

Definition 4. The algebra of stochastic generalized functions is defined as

$$
\mathbf{G}=G_{\mathbf{e}^{\prime}} / G_{\mathbf{e}}
$$

The elements of $\mathbf{G}$ are called stochastic generalized functions.

Let $\left(\varphi_{m}\right) \in G_{\mathbf{e}^{\prime}}$ we will denote by $\left[\varphi_{m}\right]$ the equivalent class $\left(\varphi_{m}\right)+G_{\mathbf{e}}$. 
Proposition 5. Let $\iota: \mathcal{G}^{*} \rightarrow \mathbf{G}$ be the application

$$
\iota(F)=\left[F_{m}\right] .
$$

Then $\iota$ is a linear embedding. Moreover, we have that for all $\varphi \in \mathcal{G}$,

$$
\iota(\varphi)=[\varphi] .
$$

Proof. It is clear from the above Proposition, that $\iota$ is a well defined linear application. Let $F \in \mathcal{G}^{*}$ such that $\iota(F)=0$. As $\left(F_{m}\right) \in G_{\mathbf{e}}$ we have $\lim _{m \rightarrow \infty}\left\|F_{m}\right\|_{p}=0$, for all $p \in \mathbb{N}_{0}$. This is the sequence $\left(F_{m}\right)$ converge weakly to 0 , it follows that $F=0$.

It remains to prove that $\iota(\varphi)=[\varphi]$, for all $\varphi \in \mathcal{G}$. Let $\varphi=\sum_{n=0}^{\infty} I_{n}\left(f_{n}\right) \in$ $\mathcal{G}$ and $p \in \mathbb{N}_{0}$. Then

$$
\begin{aligned}
e^{2 a m}\left\|\varphi-\Pi_{m} \varphi\right\|_{p}^{2} & =e^{2 a m} \sum_{n=m+1}^{\infty} n ! e^{2 p n}\left|f_{n}\right|_{0}^{2} \\
& \leq \sum_{n=m+1}^{\infty} n ! e^{2 n(p+a)}\left|f_{n}\right|_{0}^{2}
\end{aligned}
$$

for all $a \in \mathbb{N}_{0}$.

Since $\varphi \in \mathcal{G}$, we have

$$
\sum_{n=m+1}^{\infty} n ! e^{2 n(p+a)}\left|f_{n}\right|_{0}^{2}<\infty
$$

Combining these facts, we have that

$$
\lim _{m \rightarrow \infty} e^{2 a m}\left\|\varphi-\Pi_{m} \varphi\right\|_{p}^{2}=0
$$

for all $a \in \mathbb{N}_{0}$. This shows that $\left(\varphi-\Pi_{m} \varphi\right) \in G_{\mathbf{e}}$, which completes the proof.

Corollary 1. Let $\varphi, \psi \in \mathcal{G}$. Then

$$
\iota(\varphi \psi)=\iota(\varphi) \cdot \iota(\psi) .
$$

Proof. We first observe that

$$
(\varphi \psi)-\left(\varphi_{m}\right) \cdot\left(\psi_{m}\right)=(\varphi) \cdot\left(\psi-\psi_{m}\right)+\left(\varphi-\varphi_{m}\right) \cdot(\psi) .
$$

Applying Proposition 5 and Lemma 1 we obtain $(\varphi \psi)-\left(\varphi_{m}\right) \cdot\left(\psi_{m}\right) \in$ $G_{\mathrm{e}}$. Therefore $\iota(\varphi \psi)=\iota(\varphi) \cdot \iota(\psi)$.

Now, we give some examples of stochastic generalized functions. 
Example 2. The white noise generalized function is defined by

$$
W_{x}=\left[W_{m}(x)\right]=\left[I_{1}\left(\sum_{j=0}^{m} \eta_{j}(x) \eta_{j}(\cdot)\right)\right] .
$$

For $d=1$ we have the following identity

$$
\sum_{j=0}^{n} \eta_{j}(t) \eta_{j}(x)=\frac{\sqrt{n+1}}{x-t}\left(\eta_{n+1}(x) \eta_{n}(t)-\eta_{n+1}(t) \eta_{n}(x)\right) .
$$

Thus

$$
W_{x}=\left[\int \frac{\sqrt{n+1}}{x-t}\left(\eta_{n+1}(x) \eta_{n}(t)-\eta_{n+1}(t) \eta_{n}(x)\right) d B(t)\right] .
$$

Example 3. The element $W_{x}^{2}$. From the above example we have

$$
W_{x}^{2}=\left[W_{m}(x)^{2}\right]=\left[I_{1}\left(\sum_{j=0}^{m} \eta_{j}(x) \eta_{j}(\cdot)\right) I_{1}\left(\sum_{j=0}^{m} \eta_{j}(x) \eta_{j}(\cdot)\right)\right] .
$$

From the formula (10) we get

$$
W_{x}^{2}=\left[W_{m}(x) \diamond W_{m}(x)+\sum_{j=0}^{m} \eta_{j}^{2}(x)\right] .
$$

Example 4. Let $x \in \mathbb{R}$ and $t>0$, the Donsker delta is the stochastic generalized function defined by

$$
\iota\left(\delta_{x}\left(B_{t}\right)\right)=\left[\sum_{n=0}^{m} \frac{1}{n !} t^{-n} H_{n, t}(x) H_{n, t}\left(B_{t}\right)\right],
$$

where $H_{n, t}$ is the $n$-th Hermite polynomial with variance $t$ (see for instance [14]).

Definition 5. Let $\left[\varphi_{m}\right]$ and $\left[\psi_{m}\right]$ be stochastic generalized functions. We say that $\left[\varphi_{m}\right]$ and $\left[\psi_{m}\right]$ are associated, denoted by $\left[\varphi_{m}\right] \approx\left[\psi_{m}\right]$, if for all $\varphi \in \mathcal{G}$ we have that

$$
\lim _{m \rightarrow \infty} \ll \varphi_{m}-\psi_{m}, \varphi \gg=0 .
$$

We observe that the relation $\approx$ is well defined as an equivalence relation on $\mathbf{G}$.

Lemma 2. Let $F$ and $G$ be in $\mathcal{G}^{*}$. Then:

(1) If $\iota(F) \approx \iota(G)$ then $F=G$.

(2) If there exists $F \bullet G$ then $\iota(F) \cdot \iota(G) \approx \iota(F \bullet G)$.

(3) If $\psi \in \mathcal{G}$ and $F \in \mathcal{G}^{*}$ then $\iota(\psi) \iota(F) \approx \psi F$. 
Proof. (1) Combining Proposition 1 and $\iota(F) \approx \iota(G)$ we conclude that,

$$
\ll F-G, \varphi \gg=\lim _{m \rightarrow \infty} \ll\left(\Pi_{m} F-\Pi_{m} G\right) \varphi \gg=0
$$

for all $\varphi \in \mathcal{G}$. Thus $F=G$.

The proofs of (2) and (3) are straightforward.

Remark 1. We observe that our construction of stochastic generalized functions also can be done for others space of test functions and distributions spaces, for instance Hida and Kondratiev spaces.

We introduce the generalized sequences ring $\mathbf{g}$ with the purpose of to define the expectation of generalized functions of $\mathbf{G}$.

Definition 6. The generalized sequences ring, denoted by $\mathbf{g}$, is define to be

$$
\mathrm{g}=\mathbf{e}^{\prime} / \mathbf{e} .
$$

Let $\left(b_{n}\right) \in \mathbf{e}^{\prime}$ we will use $\left[b_{n}\right]$ by denoted the equivalent class $\left(b_{n}\right)+\mathbf{e}$.

It is clear from the definition that $\mathbf{e}^{\prime}$ is a ring but is not a field. In fact, $\mathbf{e}^{\prime}$ has zero divisors.

Lemma 3. a) Let $\iota_{0}: \mathbb{R} \rightarrow \mathbf{g}$ be the application

$$
\iota_{0}(a)=[a] .
$$

Then $\iota_{0}$ is an embedding.

b) $\mathbf{G}$ is a $\mathbf{g}$-module with the natural operations.

Proof. The proof is straightforward.

We define the notion of association in $\mathbf{g}$.

Definition 7. Let $\left[a_{n}\right]$ and $\left[b_{n}\right]$ be in $\mathbf{g}$. We says that $\left[a_{n}\right]$ and $\left[b_{n}\right]$ are associated, denoted by $\left[a_{n}\right] \approx\left[b_{n}\right]$, if

$$
\lim _{n \rightarrow \infty}\left(a_{n}-b_{n}\right)=0 \text {. }
$$

We observe that the relation $\approx$ is well defined equivalence relation on g.

Proposition 6. Let $\left[\varphi_{m}\right]=\left[\psi_{m}\right] \in \mathbf{G}$. Then $\left[\mathbb{E}\left(\varphi_{m}\right)\right]=\left[\mathbb{E}\left(\psi_{m}\right)\right] \in \mathbf{g}$.

Proof. Let $\left[\varphi_{m}\right] \in G$. By definition, $\left(\varphi_{m}\right) \in G_{\mathrm{e}^{\prime}}$. Thus for all $p \in \mathbb{N}_{0}$ we have that $\left(\left\|\varphi_{m}\right\|_{p}\right) \in \mathbf{e}^{\prime}$. It follows that for all $p \in \mathbb{N}_{0}$ there exists given $C>0$ and $a \in \mathbb{N}_{0}$ such that

$$
\left\|\varphi_{m}\right\|_{p}^{2} \leq C^{2} e^{2 a m}
$$

for all $m \in \mathbb{N}_{0}$. 
By definition of $\|\cdot\|_{p}$, we have that $\left|\mathbb{E}\left(\varphi_{m}\right)\right|^{2} \leq\left\|\varphi_{m}\right\|_{p}^{2}$. Thus

$$
\left|\mathbb{E}\left(\varphi_{m}\right)\right| \leq C e^{a m}
$$

for all $m \in \mathbb{N}_{0}$. This implies that $\left[\mathbb{E}\left(\varphi_{m}\right)\right] \in \mathbf{g}$.

It remains to prove the independence of the representative. By definition, $\left(\varphi_{m}-\psi_{m}\right) \in G_{\mathbf{e}}$. Thus for all $p \in \mathbb{N}_{0}$ we have that $\left(\left\|\varphi_{m}-\psi_{m}\right\|_{p}\right) \in$ e. It follows that given $a, p \in \mathbb{N}_{0}$ and $\varepsilon>0$ there exists $m_{0} \in \mathbb{N}_{0}$ such that

$$
e^{2 a m}\left\|\varphi_{m}-\psi_{m}\right\|_{p}^{2}<\varepsilon^{2}
$$

for all $m \geq m_{0}$.

By the definition of $\|\cdot\|_{p}$, we have that $\left|\mathbb{E}\left(\varphi_{m}\right)-\mathbb{E}\left(\psi_{m}\right)\right|^{2} \leq\left\|\varphi_{m}-\psi_{m}\right\|_{p}^{2}$. Thus

$$
e^{a m}\left|\mathbb{E}\left(\varphi_{m}\right)-\mathbb{E}\left(\psi_{m}\right)\right|<\varepsilon
$$

for all $m \geq m_{0}$. We conclude that $\left[\mathbb{E}\left(\varphi_{m}\right)\right]=\left[\mathbb{E}\left(\psi_{m}\right)\right]$.

Definition 8. Let $F=\left[\varphi_{m}\right]$ be a generalized function in $\mathbf{G}$. The expectation of $F$, denoted by $\mathbb{E}(F)$, is defined to be the generalized number

$$
\mathbb{E}(F)=\left[\mathbb{E}\left(\varphi_{m}\right]\right.
$$

Proposition 7. Let $f \in L^{1}(\mu)$ and $F=\left[\varphi_{m}\right] \in \mathbf{G}$ such that $F \approx f$. Then

$$
\iota_{0}(\mathbb{E}(f))=\mathbb{E}(F) .
$$

Proof. The proof is straightforward from the definitions.

\section{A Stochastic Cauchy Problem}

In this section we study the stochastic Cauchy problem

$$
\left\{\begin{array}{l}
u_{t}=L u+u W(t, x) \\
u_{0}=f
\end{array}\right.
$$

where $L$ is an uniformly elliptic partial differential operator, $f \in C_{b}^{2}\left(\mathbb{R}^{l}\right)$ and $W$ is the white noise parametric generalized function defined below in Example 5 .

In order to solve (19) we introduce the algebra $G^{\alpha}(D)$ of parametric generalized functions. We proceed in a similar way to the construction of the algebra $G$, the details are left to the reader.

Let $D$ a domain in $\mathbb{R}^{d}$ and $\alpha \in \mathbb{N}_{0}^{d}$. We denote by $C^{\alpha}(D, \mathcal{G})$ the set of functions $f: D \times \mathcal{S}^{\prime}\left(\mathbb{R}^{d}\right) \rightarrow \mathbb{R}(\mathbb{C})$ such that $f(x, \cdot) \in \mathcal{G}$ for all $x \in D$ and $f(\cdot, \omega) \in C_{b}^{\alpha}(D)$ for almost $\omega \in \mathcal{S}^{\prime}\left(\mathbb{R}^{d}\right)$. 
Definition 9. Let $G_{\mathbf{e}^{\prime}}^{\alpha}(D)$ be the set of $\left(f_{m}\right) \in\left(C^{\alpha}(D, \mathcal{G})\right)^{\mathbb{N}_{0}}$ such that

$$
\left(\sup _{x}\left\|D^{\beta} f_{m}(x, \cdot)\right\|_{p}\right) \in \mathbf{e}^{\prime}
$$

for all $\beta \in \mathbb{N}_{0}^{d}$ with $\beta \leq \alpha$ and $p \in \mathbb{N}_{0}$.

We observe that $G_{\mathbf{e}^{\prime}}^{\alpha}(D)$ is a subalgebra of $\left(C^{\alpha}(D, \mathcal{G})\right)^{\mathbb{N}_{0}}$ and for $\gamma \in \mathbb{N}_{0}^{d}$, $0 \leq \gamma \leq \alpha$ the partial differential operator $D^{\gamma}$ transform $G_{\mathbf{e}^{\prime}}^{\alpha}(D)$ into $G_{\mathbf{e}^{\prime}}^{\alpha-\gamma}(D)$.

Definition 10. Let $G_{\mathbf{e}}^{\alpha}(D)$ be the set of $\left(f_{m}\right) \in\left(C^{\alpha}(D, \mathcal{G})\right)^{\mathbb{N}_{0}}$ such that

$$
\left(\sup _{x}\left\|D^{\beta} f_{m}(x, \cdot)\right\|_{p}\right) \in \mathbf{e}
$$

for all $\beta \in \mathbb{N}_{0}^{d}$ with $\beta \leq \alpha$ and $p \in \mathbb{N}_{0}$.

It is clear that $G_{\mathbf{e}}^{\alpha}(D)$ is an ideal of $G_{\mathbf{e}^{\prime}}^{\alpha}(D)$.

Definition 11. We define the algebra of parametric generalized functions $G^{\alpha}(D)$ as

$$
G_{\mathbf{e}^{\prime}}^{\alpha}(D) / G_{\mathbf{e}}^{\alpha}(D)
$$

The elements of $G^{\alpha}(D)$ are called parametric generalized functions.

In the case that $\alpha=(0, . ., 0)$ we denote $G^{\alpha}(D)$ by $G(D)$. It is clear that $\mathbf{G} \subset G^{\alpha}(D)$ and $C_{b}^{\alpha}(D) \subset G^{\alpha}(D)$.

Example 5. Let $D$ be a domain in $\mathbb{R}^{d}$ and $\alpha \in \mathbb{N}_{0}^{d}$. The white noise parametric generalized function is defined by

$$
W_{x}=\left(W_{m}(x)\right)=\left(I_{1}\left(\sum_{j=0}^{m} \eta_{j}(x) \eta_{j}(\cdot)\right)\right),
$$

for $x \in D$.

In fact, we observe that there exists constants $C_{\alpha}$ and $a_{\alpha}$ such that for all $0 \leq \beta \leq \alpha$,

$$
\begin{aligned}
\left\|D_{x}^{\beta} W_{m}(x)\right\|_{p}^{2} & =\left\|D_{x}^{\beta} W_{m}(x)\right\|_{0}^{2} \\
& =\left\|I_{1}\left(\sum_{j=0}^{m} D_{x}^{\beta} \eta_{j}(x) \eta_{j}(\cdot)\right)\right\|_{0}^{2} \\
& =\left|\sum_{j=0}^{m} D_{x}^{\beta} \eta_{j}(x) \eta_{j}(\cdot)\right|_{0}^{2} \\
& =\sum_{j=0}^{m}\left|D_{x}^{\beta} \eta_{j}(x)\right|^{2} \\
& \leq C_{\alpha}(m+1)^{a_{\alpha}} .
\end{aligned}
$$


Definition 12. We says that $u=\left[u_{m}\right] \in G^{(1,2, \ldots, 2)}\left([0, T] \times \mathbb{R}^{l}\right)$ is a generalized solution of the Cauchy problem (19) if $u_{t}=L u+u W(t, x)$ in $G\left([0, T] \times \mathbb{R}^{l}\right)$ and $u_{0}=f$ in $G^{(2, . ., 2)}\left(\mathbb{R}^{l}\right)$.

We observe that $\left[u_{m}\right] \in G^{\alpha}(D)$ implies that $\left[u_{m}(0, \cdot)\right]$ is a well defined element of $G^{(2, . ., 2)}\left(\mathbb{R}^{l}\right)$.

Theorem 2. There exists an unique generalized solution $u=\left[u_{m}\right]$ in $G^{\alpha}(D)$ for the Cauchy problem (19).

Proof. Let $d=1+l, \alpha=(1,2, \ldots, 2) \in \mathbb{N}_{0}^{1+l}$ and $D=[0, T] \times \mathbb{R}^{l}$ $(T>0)$. We consider that $L$ have the form

$$
L f=\frac{1}{2} \sum_{i, j=1}^{l} a_{i j} \frac{\partial^{2}}{\partial x_{i} \partial x_{j}} f+\sum_{i=1}^{l} b_{i} \frac{\partial}{\partial x_{i}} f
$$

where $a_{i j}$ and $b_{j}$ belong to $C_{b}^{2}\left(\mathbb{R}^{l}\right)$ for all $i$ and $j$.

We split the proof in three steps:

(a) We solve the family of parabolic problems $\left(m \in \mathbb{N}_{0}\right)$

$$
\frac{d}{d t} u_{m}=L u_{m}+u_{m} W_{m}(t, x)
$$

with $u(x, 0)=f(x)$.

(b) We check that the nets of solutions $\left(u_{m}\right)$ belongs to $G_{\mathrm{e}^{\prime}}^{\alpha}(D)$.

(c) We check that if $\left(u_{m}\right)$ and $\left(v_{m}\right)$ are two nets of solutions of (19), then $\left[u_{m}\right]=\left[v_{m}\right]$.

In order to prove (a), we use that the Feymann-Kac formula give a representation of the solution of (201) (see for instance [11]). We observe that there exist $\sigma=\left(\sigma_{i j}\right) \in C^{2}\left(\mathbb{R}^{l} ; \mathbb{R}^{l \times l}\right)$ such that

$$
a_{i j}=\sum_{k=1}^{l} \sigma_{i k} \sigma_{j k} .
$$

We consider the following stochastic differential equation,

$$
d X=\sigma(X) d B+b(X) d t,
$$

where $B$ is an $l$-dimensional Brownian motion in an auxiliar probability space, $\sigma=\left(\sigma_{i j}\right)$ and $b=\left(b_{i}\right)$.

The solution of (21) with $X(0)=x \in \mathbb{R}^{l}$ is denoted by $X(t, x)$. Applying the Feynman-Kac formula to $u_{m}$ we have

$$
u_{m}(t, x)=\mathbb{E}\left(f(X(t, x)) e^{\int_{0}^{t} W_{m}(t-s, X(s, x)) d s}\right),
$$

where $\mathbb{E}$ denotes the expectation in the auxiliar probability space.

It remains to prove b), this is $\left(u_{m}\right)$ belongs to $G_{\mathbf{e}^{\prime}}^{\alpha}(D)$. 
We claim that for each $p \in \mathbb{N}_{0}$ there exists constants $C$ and $a$ such that

$$
\sup _{(t, x)}\left\|u_{m}(t, x)\right\|_{p}, \sup _{(t, x)}\left\|\frac{d}{d t} u_{m}(t, x)\right\|_{p}, \sup _{(t, x)}\left\|D^{\beta} u_{m}(t, x)\right\|_{p} \leq C e^{a m}
$$

where $0 \leq \beta \leq(2, \ldots, 2)$.

In fact, by the definition of Wick exponential (7),

$$
u_{m}(t, x)=\mathbb{E}\left(f(X(t, x)) e^{\frac{1}{2}\left|\int_{0}^{t} \sum_{j=0}^{m} \eta_{j}((t-s), X(s, x)) \eta_{j}(\cdot) d s\right|_{0}^{2}} e^{: \int_{0}^{t} W_{m}(t-s, X(s, x)) d s}\right) .
$$

From the uniform boundedness and orthonormality of the Hermite functions, we have that there exist a positive constant $a$ depending of $T$ such that

$$
\left|\int_{0}^{t} \sum_{j=0}^{m} \eta_{j}((t-s), X(s, x)) \eta_{j}(\cdot) d s\right|_{0}^{2} \leq a m .
$$

Combining (23),$(24)$ and (8) , we obtain that $\sup _{(t, x)}\left\|u_{m}(t, x)\right\|_{p}^{2}$ is bounded by

$$
\left(\sup _{x \in \mathbb{R}^{l}}|f(x)|\right)^{2} e^{a m} .
$$

In order to show that $\sup _{(t, x)}\left\|\frac{d}{d t} u_{m}(t, x)\right\|_{p} \leq C e^{a m}$, applying the Itô formula in (22), we obtain that

$$
\begin{aligned}
\frac{d}{d t} u_{m}(t, x)= & \mathbb{E}\left(\left\{f(X(t, x))\left(W_{m}(0, X(t, x))+\int_{0}^{t} \frac{d}{d t} W_{m}(t-s, X(s, x)) d s\right)\right.\right. \\
& \left.\left.+L f_{m}(X(t, x))\right\} e^{\int_{0}^{t} W_{m}(t-s, X(s, x)) d s}\right) .
\end{aligned}
$$

We need dominate the following terms:

a) $A_{1}=\| \mathbb{E}\left(f(X(t, x)) W_{m}(0, X(t, x)) e^{\int_{0}^{t} W_{m}(t-s, X(s, x)) d s} \|_{p}^{2}\right.$,

b) $A_{2}=\| \mathbb{E}\left(f(X(t, x)) \int_{0}^{t} \frac{d}{d t} W_{m}(t-s, X(s, x)) d s e^{\int_{0}^{t} W_{m}(t-s, X(s, x)) d s} \|_{p}^{2}\right.$,

c) $A_{3}=\| \mathbb{E}\left(L f(X(t, x)) e^{e_{0}^{t} W_{m}(t-s, X(s, x)) d s} \|_{p}^{2}\right.$.

a) We observe that $A_{1}$ is equal to

$$
\| \mathbb{E}\left(f(X(t, x)) e^{\frac{1}{2}\left|\int_{0}^{t} \sum_{j=0}^{m} \eta_{j}((t-s), X(s, x)) \eta_{j}(\cdot) d s\right|_{0}^{2}} W_{m}(0, X(t, x)) e^{: \int_{0}^{t} W_{m}(t-s, X(s, x)) d s} \|_{p}^{2} .\right.
$$

By the boundeness of $f$ and the inequality (24) we have that

$$
A_{1} \leq C e^{a m} \mathbb{E}\left(\left\|W_{m}(0, X(t, x)) e^{: \int_{0}^{t} W_{m}(t-s, X(s, x)) d s}\right\|_{p}^{2}\right) .
$$

From (91) there exists $C_{p}>0$ and $r, r^{\prime} \in \mathbb{N}_{0}$ such that

$$
\left\|W_{m}(0, X(t, x)) e^{: \int_{0}^{t} W_{m}(t-s, X(s, x)) d s}\right\|_{p}^{2}
$$

is lower or equal to

$$
C_{p}\left\|W_{m}(0, X(t, x))\right\|_{r}^{2}\left\|e^{: \int_{0}^{t} W_{m}(t-s, X(s, x)) d s}\right\|_{r^{\prime}}^{2} \leq C e^{a m} .
$$


Thus $A_{1} \leq C e^{a m}$.

b) and c). The estimative for $A_{2}$ is obtained in a similar way and the estimative for $A_{3}$ is analogous to the estimative for $\left\|u_{m}\right\|_{p}^{2}$. We conclude that

$$
\left\|\frac{d}{d t} u_{m}(t, x)\right\|_{p} \leq C e^{a m}
$$

It remains to prove that $\sup _{(t, x) \in K}\left\|D^{\beta} u_{m}(t, x)\right\|_{p} \leq C e^{a m}$ for $0 \leq \beta \leq$ $(2, \ldots, 2)$.

We first observe that $D^{i} u_{m}(i=1, . ., l)$ is equal to

$$
\begin{gathered}
\mathbb{E}\left(\left\{\sum_{j=1}^{l} D^{i} f(X(t, x)) D^{i} X_{j}(t, x)+f(X(t, x)) \sum_{j=1}^{l} \int_{0}^{t} D^{i} W_{m}(t-s, X(t, x))\right.\right. \\
\left.\left.\left.\times D^{i} X_{j}(s, x)\right) d s\right\} e^{\int_{0}^{t} W_{m}(t-s, X(s, x)) d s}\right) .
\end{gathered}
$$

It is clear that $D_{i} X_{j}$ satisfy the following system of linear stochastic equations

$$
\begin{aligned}
D^{i} X_{j}=\delta_{i j} & +\int_{0}^{t} \sum_{k, h=1}^{l}\left(D^{k} \sigma_{j h}\right)(X(s, x)) D^{i} X_{k}(s, x) d B_{h}(s)+ \\
& +\int_{0}^{t} \sum_{k=1}^{l}\left(D^{k} b_{j}\right)(X(s, x)) D^{i} X_{k}(s, x) d s .
\end{aligned}
$$

From the equation (25) and Gronwall lemma we get that

$$
\mathbb{E}\left(\sum_{i=1}^{l}\left|D^{i} X_{j}(t, x)\right|^{2}\right)
$$

is locally uniformly bounded in $t$ and $x$.

We need dominate the terms

d) $B_{1}=\left\|\mathbb{E}\left(\sum_{j=1}^{l} D^{i} f(X(t, x)) D^{i} X_{j}(t, x) e^{\int_{0}^{t} W_{m}(t-s, X(s, x)) d s}\right)\right\|_{p}^{2}$,

e) $\left.B_{2}=\| \mathbb{E}\left(f(X(t, x))\left(\sum_{j=1}^{l} \int_{0}^{t} D^{i} W_{m}(t-s, X(t, x))\right) D^{i} X_{j}(s, x)\right) d s\right)$

$$
e^{\int_{0}^{t} W_{m}(t-s, X(s, x)) d s} \|_{p}^{2} .
$$

d) We observe that $B_{1}$ is equal to

$$
\| \mathbb{E}\left(\sum_{j=1}^{l} D^{i} f(X(t, x)) D^{i} X_{j}(t, x) e^{: \int_{0}^{t} W_{m}(t-s, X(s, x)) d s} e^{\left.\frac{1}{2}\left|\int_{0}^{t} \sum_{j=0}^{m} \eta_{j}((t-s), X(s, x)) \eta_{j}(\cdot) d s\right|_{0}^{2}\right)} \|_{p}^{2} .\right.
$$


By the boundeness of the derivatives of $f$ and the inequality (24) we have that

$$
B_{2} \leq C e^{a m} \mathbb{E}\left(\sum_{i=1}^{l}\left|D^{i} X_{j}(t, x)\right|^{2}\left\|e^{: \int_{0}^{t} W_{m}(t-s, X(s, x)) d s}\right\|_{p}^{2}\right) \leq C e^{a m} .
$$

e) The estimative for $B_{2}$ is obtained in an analogous way, using (9). Therefore,

$$
\sup _{(t, x)}\left\|D^{i} u_{m}(t, x)\right\|_{p} \leq C e^{a m} .
$$

The estimative for $\left\|D^{i} D^{j} u_{m}\right\|_{p}$ is done in a similar way. So we conclude that $\left[u_{m}\right] \in G^{\alpha}(D)$.

We considerer the uniqueness. Suppose that $\left[u_{m}\right]$ and $\left[v_{m}\right]$ are two generalized solutions of (19), we denote by $d_{m}$ to $u_{m}-v_{m}$. By the definition, we have that $d_{m}$ satisfy

$$
\left\{\begin{array}{l}
\frac{d}{d t} d_{m}=L d_{m}+d_{m} W_{m}(t, x)+N_{m}(t, x) \\
u_{m}(0, x)=N_{0, m}(x)
\end{array}\right.
$$

with $N_{m}(t, x) \in G_{\mathbf{e}}^{\alpha}(D)$ and $N_{0, m} \in G_{\mathbf{e}}^{\alpha}\left(\mathbb{R}^{l}\right)$. Applying the FeynmanKac formula to $d_{m}$ we obtain that

$$
\begin{aligned}
d_{m}(t, x)= & \mathbb{E}\left(N_{0, m}(X(t, x)) e^{\int_{0}^{t} W_{m}(t-s, X(s, x)) d s}\right. \\
& \left.+\int_{0}^{t} N_{m}(s, x) e^{\int_{0}^{s} W_{m}(s-u, X(u, x)) d u} d s\right) .
\end{aligned}
$$

It is straightforward using similar techniques that

$$
\sup _{(t, x)}\left\|d_{m}(t, x)\right\|_{p}, \sup _{(t, x)}\left\|\frac{d}{d t} d_{m}(t, x)\right\|_{p}, \sup _{(t, x)}\left\|D^{\beta} d_{m}(t, x)\right\|_{p} \in \mathbf{e}
$$

for each $p \in \mathbb{N}_{0}$ and $0 \leq \beta \leq(2, \ldots, 2)$. We conclude that the solution of (19) is unique.

We end the paper with the following remarks.

Remark 2. Wick solution versus generalized solution: Let us consider the following Cauchy problem,

$$
\left\{\begin{array}{l}
v_{t}=L v+v \diamond W(t, x) \\
v_{0}=f
\end{array}\right.
$$

where $L$ is an uniformly elliptic partial differential operator, $f \in C_{b}^{2}\left(\mathbb{R}^{l}\right)$ and $W$ is the white noise parametric generalized function. 
We observe that the proof of existence and uniqueness given in [24] extends to the following Cauchy problem,

$$
\left\{\begin{array}{l}
\frac{d}{d t} v_{m}=L v_{m}+v_{m} \diamond W_{m}(t, x) \\
v_{m}(\cdot, 0)=f
\end{array}\right.
$$

where $W(t, x)=\left[W_{m}(t, x)\right]$ is the white noise parametric generalized function.

The sequence of solutions $\left(v_{m}\right)$ converges in the Hida distribution space to the solution of equation (27) (see [17]).

We have that the $S$-transformation of the solution of equation (28) is

$$
S\left(v_{m}(t, x)\right)(h)=\mathbb{E}\left(f(X(t, x)) e^{\int_{0}^{t} h_{m}(t-s, X(s, x)) d s}\right)
$$

and the S-transformation of the generalized solution $u=\left[u_{m}\right]$ of (19) is

$S\left(u_{m}(t, x)\right)(h)=\mathbb{E}\left(f(X(t, x)) e^{\int_{0}^{t} h_{m}(t-s, X(s, x)) d s}\right) e^{\left|\int_{0}^{t} W_{m}(., t-s, X(s, x)) d s\right|_{0}}$.

This implies that the generalized solution $u=\left[u_{m}\right]$ is not associated to any stochastic distribution for $d>1$.

Remark 3. We can show in a similar way that there exists an unique generalized solution of the stochastic Cauchy problem (19) with initial data $f \in G^{(2, . ., 2)}\left(\mathbb{R}^{l}\right)$.

\section{REFERENCES}

[1] K. Aase, B. Oksendal, N. Privault and J. Uboe: A white noise generalization of the Clark-Haussmann-Ocone theorem with application to mathematical finance. Finance Stoch. 4, 465-496, 2000.

[2] S. Albeverio, Z. Haba and F. Russo: A two-space dimensional semilinear heat equation perturbed by (Gaussian) white noise. Probab. Theory Related Fields 121, 3, 319-366, 2001.

[3] S. Albeverio, Z. Haba and F. Russo: On non-linear two-space-dimensional wave equation perturbed by space-time white noise. Israel Math. Conf. Proc. 10, 1-25, 1996.

[4] P. Antosik, J. Mikusiński and R. Sikorski: Theory of Distributions. The sequential approach. Elsevier Scientific Publishing Company, 1973.

[5] F. Benth and J. Potthoff: On the martingale property for generalized stochastic process. Stochastics Stochastics Rep. 58 , 349-367, 1996.

[6] F. Benth and T. Theting: Some regularity results for the stochastic pressure equation of Wick-type. Stochastic Anal. Appl. 20, 6, 1191-1223, 2002.

[7] H. A. Biagioni: A nonlinear theory of generalized functions. Lect. Notes Math. 1421. Springer-Verlag, Berlin, 1990.

[8] J. F. Colombeau: Elementary introduction to new generalized functions. Noth-Holland Math Studies 113, Noth Holland, Amsterdam, 1985.

[9] P. Catuogno, S. Molina and C. Olivera: On Hermite representation of distributions and products. Integral Transforms and Special Functions 18, 4, 233-243, 2007. 
[10] P. Chow: Generalized solution of some parabolic equations with a random drift. Appl. Math. Optim, 20, 1, 1-17, 1989.

[11] M. Freidlin: Functional integration and partial differential equations. Princeton University Press, 1985.

[12] M. Grosser , M. Kunzinger and M. Oberguggenberger and R. Steinbauer: Geometric theory of generalized function with applications to general relativity. Kluwer Academic Publishers, Dordrecht, 2001.

[13] M. Grothaus, Y.G. Kondratiev and L. Streit: Regular generalized functions in Gaussian analysis. Infinite Dimensional Anal. Quantum Prob. 2, 1, 1-25. 1999.

[14] T. Hida, H. Kuo, J. Potthoff and L. Streit: White noise. An infinitedimensional calculus. Kluwer, 1993.

[15] H. Holden, B. Oksendal, J. Uboe and T. Zhang: Stochastic partial differential equations. A modeling, white noise functional approach. Birkhuser Boston, 1996.

[16] Z. Huang and J. Yan: Introduction to infinite dimensional stochastic analysis. Kluwer, 1999.

[17] T. Lindstrom, B. Oksendal, J. Uboe and T. Zhang: Stability properties of stochastic partial differential equations. Stochastic Anal. Appl. 13, 2, 177204, 1995.

[18] H. Kuo: White noise distribution theory. CRC, 1996.

[19] H. Kunita: Generalized solution of a stochastic partial differential equation. J. Theoretical Probability 7, 2, 279-308, 1994.

[20] S. Lototsky and B. Rozovskii: Wiener chaos solutions of linear stochastic evolution equations. Ann. Probab. 34, 2, 638-662, 2006.

[21] D. Nualart and M. Zakai: Generalized Brownian functionals and the solution to a stochastic partial differential equation. J. Funct. Anal. 84, 2, 279-296, 1989.

[22] M. Oberguggenberger: Multiplication of distributions and applications to partial differential equations. Pitman Research Notes in Math. Series 259. Ed. Longman Science and Technology, 1993.

[23] M. Oberguggenberger and F. Russo: Nonlinear SPDEs: Colombeau solutions and pathwise limits. Stochastic analysis and related topics, VI (Geilo, 1996), 319-332, Progr. Probab., 42, Birkhauser Boston, Boston, MA, 1998.

[24] J. Potthoff, G. Vage, and H. Watanabe: Generalized solutions of linear parabolic stochastic partial differential equations. Appl. Math. Optim, 38, 1, 95-107, 1998.

[25] J. Potthoff and M. Timpel, On a dual pair of spaces of smooth and generalized random variables. Potential Anal. 4, 6, 637-654, 1995.

[26] F. Russo, Colombeau generalized functions and stochastic analysis, Edit. A.l. Cardoso, M. de Faria, J Potthoff, R. Seneor, L. Streit, Stochastic analysis and applications in physics, NATO Adv. Sci. Inst. Ser. C Math. Phys. Sci., Kluwer Acad. Publ., Dordrecht, 449(1994) 329-249.

[27] L. Schwartz: Théorie des distributions. Hermann, Paris, 1966.

[28] B. Simon and M. Reed: Methods of modern mathematical physics. vol. 1. Academic Press, 1980.

[29] M. Valdivia: Topics in locally convex spaces. North-Holland Mathematics Studies, 67. Notas de Matemtica 85. North-Holland Publishing Co., 1982. 
[30] J. B. Walsh: An introduction to stochastic partial differential equations, In: Ecole dEte de Probabilites de Saint Flour XIV, Lecture Notes in Mathematics 1180 (1986) 265-438.

Departamento de Matemática, Universidade Estadual de Campinas,, 13.081-970 - CAMPINAS - SP, BRAZIL.

E-mail address: pedrojc@ime.unicamp.br ; colivera@ime.unicamp.br 